\title{
Selection of Phase Space Reconstruction Parameters for EMG Signals of the Uterus
}

\author{
Ewelina Brzozowska ${ }^{1}$, Marta Borowska ${ }^{1}$ \\ 1 Department of Materials and Biomedical Engineering, Bialystok University of Technol- \\ ogy, Poland
}

\begin{abstract}
Biological time series have a finite number of samples with noise included in them. Because of this fact, it is not possible to reconstruct phase space in an ideal manner. One kind of biomedical signals are electrohisterographical (EHG) datasets, which represent uterine muscle contractile activity. In the process of phase space reconstruction, the most important thing is suitable choice of the method for calculating the time delay $\tau$ and embedding dimension $d$, which will reliably reconstruct the original signal. The parameters used in digital signal processing are key to arranging adequate parameters of the analysed attractor embedded in the phase space. The aim of this paper is to present a method employed for phase space reconstruction for EHG signals that will make it possible for their further analysis to be carried out.
\end{abstract}

\section{Introduction}

Information about the complexity of a dynamic system is difficult to obtain, but with help in solving that problem comes nonlinear dynamics (deterministic chaos). Dynamic systems could be described by linear and non-linear differential equations. The majority of systems which occur in nature and medicine are non-linear, so they are "responsive" to the initial conditions. For this reason, small changes in them cause significant differences in the signal at the output of the system. Non-stationary from their nature, biomedical signals could be analysed by linear and non-linear methods. However, this also shows that non-linear methods are more useful to analyse the dynamics of a process. During the analysis of such dynamic biological systems, often the non-linear part of the signal is ignored. That problem is solved more easily by linear methods, which allow the description of the system composed of several variables interacting with each other to be taken as the analytical solution. 
In the analysis of dynamical systems, the first step is usually the reconstruction of the attractor in phase space. The attractor is a set of points in phase space (nonlinear dynamic system description), for which asymptotically tends the trajectories over the time close to infinity (Ruelle, 1990). In processing the structure of most biomedical signals, an effective method for visualization of the attractor is calculation of the time delay and embedding dimension (Erem et al., 2016).

Reconstruction of the phase space in cases where the equations describing such a system exist, or in which knowledge of all of the system's variables is associated with a particular system, is simple. However, in the case of ignorance of a system's variables, so-called "phase space reconstruction" can be performed (Huffaker, 2010).

The method of reconstruction in this d-dimensional phase space has been proposed by Takens (1981) and Packard et al. (1980). They had to have only one variable $x$ measured $N$ times with the sampling frequency $f_{s}=1 / t_{s}$.

$\mathrm{D}$ - dimensional phase space consists of d-independent variables. With a one-dimensional time series, other dimensions are obtained by delayed counterparts of the observed variable. For the time series matched as $\left\{x_{1}, x_{2}, \ldots, x_{N}\right\}$ there are vectors described as:

$$
Y_{i}=\left[\begin{array}{llll}
x_{i} & x_{i+\tau} & \cdots & x_{i+(d-1) \tau}
\end{array}\right],
$$

where $\tau=i t_{s}$ is the reconstruction of delay or lag, $i=1,2, \ldots, N-(d-1) \tau$ and $d$ - the embedding dimension.

\section{Methods of Selecting Time Delay}

The attractor reconstruction method of Takens (1981) requires the proper selection of time delay $\tau$. For small values of $\tau$ the coordinates of vectors $Y_{i}$ are themselves nearly equal. The reconstructed vectors in phase space are too close to each other, making it impossible to obtain information on the dynamics of the system (redundance). If the time delay $\tau$ is too large, the reconstructed vectors in phase space are far apart. At the same time, the samples come out of the scope of correlation, which is not very high for dynamic systems (irrelevance) (Casdagli et al., 1991). Practically biological time series have a finite number of samples (including noise), so the attractor reconstructed phase space is not ideal. That is why the method for determining the time delay $\tau$ is so important in the original signals' phase space reconstruction (Kliková et al., 2011). In the literature, several methods by 
which value $\tau$ can be selected are mentioned. These include: autocorrelation, mutual information, higher order statistics, fill factor and wavering product. It is well known from research on EHG signals that the most valuable measure of time delay is mutual information (Alamedine et al., 2014; Diab et al., 2015; Przybyła et al., 2014).

Mutual information $I(\tau)$ was developed by Fraser et al. (1986). This method can measure the statistical independence of the points $x(k)$ and $x(k+\tau)$. Average mutual information can be defined by the formula (Abarbanel et al., 1993):

$$
I(\tau)=\sum_{k} P(x(k), x(k+\tau)) \log _{2} \frac{P(x(k), x(k+\tau))}{P(x(k)) \cdot P(x(k+\tau))},
$$

where $P(x(k)), P(x(k+\tau))$ are probability distributions and $P(x(k), x(k+\tau))$ is the cumulative probability distribution. First, the minimum of mutual information is used as an indicator of time delay (Palit et al., 2015).

Estimation of the average of mutual information from experimental data is not difficult. In the first step, a histogram is created, wherein each signal value is assigned to the frequency of its occurrence. This method has found wide practical biomedical application, mostly in EEG (Ouyang et al., 2016) and for EHG (uterine EMG) signals (Diab et al., 2012).

Autocorrelation is the second criteria of choosing the time delay $\tau$; it can be associated with the autocorrelation function $R_{x x}(\tau)$ :

$$
R_{x x}=\frac{\frac{1}{N} \sum_{k=1}^{N}[x(k+\tau)-\bar{x}][x(k)-\bar{x}]}{\frac{1}{N} \sum_{k=1}^{N}[x(k)-\bar{x}]^{2}},
$$

where: $\bar{x}=\frac{1}{N} \sum_{k=1}^{N} x(k)$.

Autocorrelation estimates cross-correlation between pairs of points as a function of their distance in time. There are several choices for time delay of the autocorrelation function by reading the characteristic points of the curve, for example: the first zero of this function, the first minimum, and the first point of inflection. Choosing the right option is dependent on the dynamic system. Accepting the $\tau$ of the first zero of the function indicates that the signals $x(k)$ and $x(k+\tau)$ are linearly independent. The relationship between the spatial distribution of the reconstructed attractor points and temporal autocorrelation of the signal is not clear. In one paper on this subject, Bassingthwaighte et al. (2013) adopted for the selection of time delay a value which had an autocorrelation function reduced to $1 / e=$ 0.37. Albano et al. (1988) and Pritchard et al. (1995) used the equation: $\tau=3 t_{1 / e} /(d-1)$ where $t_{1 / e}$ is the value for which the autocorrelation function is reduced to $1 / e=0.37$ and $d$ is embedding dimension. 
Albano et al. (1991) used so-called higher-order statistics (HOS) to estimate time delay. HOS are extensions of measurement of the second order, such as the autocorrelation function or the power spectrum. For real-time series, HOS takes the higher-order moments into account. The application of HOS is usually performed using cumulants. They found that several functions have extremes in the same place, which may be a good estimate $\tau$. The HOS method is now currently used in bioelectrical EEG and EHG signal analysis (Lainscsek et al., 2015).

Fill factor is a geometric estimation of time delay which gives the maximum distance between trajectories (Buzug et al., 1992b). After the attractor reconstruction of the phase space of dimension $d$, defined as $d+1$, random points become the vertices of the hyper-parallelepiped. Then, the volume is calculated. The greater the volume of the thus formed figure, the larger the volume of space phase occupied (to be filled) by the attractor. Selecting a number of hyper-parallelepipeds can estimate their average volume. Maximum fill factor gives the value of the time delay, and its value is the same for different embedding dimensions (Korus et al., 2015). This method is not perfect, because for some attractors, fill factor shows no explicit extremes. Buzug et al. (1992a) introduced - in addition to the fill factor - integral local deformation. The essence of it is to study some topological properties of the attractor, which should not be changed during reconstruction.

The wavering product was introduced by Liebert et al. (1991), who led the topological considerations concerning neighbour relations between the points of the attractor. The wavering product is useful for the evaluation of the smallest embedding dimension because it keeps the topological in-

variant. This is achieved after recognizing that the false nearest neighbours are present when the embedding dimension is too small. The first minimum of a wavering product is the proper choice for time delay reconstruction (Piórek, 2016).

\section{Methods of Selecting Embedding Dimension}

It is very important to choose a sufficient embedding dimension in phase space, because it should completely represent the dynamics of that dynamic system. Phase space reconstruction based on Takens' method requires two variables - the time delay $\tau$ and the embedding dimension $d$ (Jaśkowski, 1995).

The criterion to select the embedding dimension is based on properties of "non-cutting" trajectory and reversibility of the reconstructed attractor's 
mapping. As stated by Takens (1981), embedding dimension $d$ should be higher than attractor dimension $D$ according to the relationship: $d \geq 2 D+1$.

There is a method proposed by Grassberger et al. (1983), which allows for simultaneous calculation of the two dimensions. Subsequently, the attractor is reconstructed in the low-dimensional phase space and its dimension, for example the correlation dimension, is calculated. There are several methods for selecting the embedding dimension. The most useful are: false nearest neighbours (FNN), singular-value decomposition (SVD) (Broomhead et al., 1989) and the Cao criterion.

The way to estimate the minimal embedding dimension is to use the false nearest neighbours (FNN) method (Abarbanel et al., 1993). The idea behind the method is the fact there are phase space points (vectors), which, in the case of a small embedding dimension, seem to be the neighbours the distance between them is very small. After enlarging space dimension, distance also increases rapidly - it means that we deal with a so called "false nearest neighbour".

All vectors have their neighbours, the nearest being $y^{N N}(k)$. The distance between $y(k)$ and $y^{N N}(k)$ is matched by $R_{d}(k)$. The euclidean distance plays a very important role. Increasing the dimension of the space by 1 gives the distance between $y(k)$ and $y^{N N}(k)$ in $(d+1)$-dimensional phase space. If the distance $R_{d+1}(k)$ is large in comparison with the $R_{d}(k)$, the vector could be considered as the nearest false neighbour. This fact is described by the criterion indicated as follows:

$$
\frac{\left|x(k+d \tau)-x^{N N}(k+d \tau)\right|}{R_{d}(k)}>R_{t d},
$$

where $R_{t d}$ is the so-called tolerance threshold. In calculation practice, the number of false nearest neighbours is approximately constant for $10 \leq R_{t d} \leq 50$.

This method has its drawbacks. If we have a time series consisting of a relatively small number of bins, we will get a very small embedding dimension, while increasing the number of samples causes its increase. In practice, we have finite data sets, so points may be close neighbours but distance does not increase with the increase of $d$ as is required.

The Cao's (1997) criterion is a very interesting method for assessing minimal embedding dimension. The procedure is similar to false nearest neighbours. For a given time series $x_{1}, x_{2}, \ldots, x_{N}$ vectors are constructed in $d$-dimensional phase space. Similar to the FNN method, there is parameter

$$
a(k, d)=\frac{\left\|y_{d+1}(k)-y_{d+1}^{N N}(k, d)\right\|}{\left\|y_{d}(k)-y_{d}^{N N}(k, d)\right\|} .
$$


In the case where $\left\|y_{d}(k)-y_{d}^{N N}(k, d)\right\|=0$, the next nearest neighbour is taken into account. Other remarks are the same as for the previously described method of FNN. Perfect choice of the embedding dimension means that there are no false nearest neighbours, but the main problem is the choice of the tolerance threshold. Cao has introduced quantity $E(d, \tau)$ described by:

$$
E(d, \tau)=\frac{1}{N-d \tau} \sum_{k=1}^{N-d \tau} a(k, d),
$$

which is the average of the all $a(k, d) . E(d, \tau)$ depends on the embedding dimension $d$ and time delay $\tau$ and is equal to $E(d)-E(d, \tau)=E(d)$, because time delays are chosen at the beginning of the calculation. To investigate the changes of magnitude during the transition from dimension $d$ to next $d+1$, it was defined:

$$
E 1(d)=\frac{E(d+1)}{E(d)}
$$

Cao found that $E 1(d)$ does not change significantly over a certain value $d 0$, if the only analysed time series comes from the attractor. Therefore, we assume $d 0$ as a value of the embedding dimension. In practice (due to the fact that the tested data have a finite length), it can be quite difficult to determine whether $E 1(d)$ does not significantly change. For this reason, another quantitative gauge has been introduced, which also allows one to distinguish deterministic signals from stochastic. It was marked as $E 2(d)$ :

where:

$$
E 2(d)=\frac{E^{*}(d+1)}{E^{*}(d)},
$$

$$
E^{*}=\frac{1}{N-d \tau} \sum_{k=1}^{N-d \tau}\left|x(k+d \tau)-x^{N N}((k, d)+d \tau)\right| .
$$

This method is now used in many fields of science (Chen et al., 2014; Xia et al., 2016).

\section{Application in Uterine Bioelectrical Activity Signal Processing - Phase Space Reconstruction}

The data used for this research was recorded at the Akureyri Primary Health Care Centre and Landspitali University Hospital in Iceland (Alexandersson et al., 2015), between 2008 and 2010. The signals used were provided as an open dataset on the Physionet web (Goldberger et al., 2000). This database consists of records of 122 EHG signals, performed on 45 pregnant women, derived from a system consisting of 16 electrodes in a 4-by- 4 
configuration on the patient's abdomen. They were divided into two groups: first, in the third trimester of pregnancy and second, during labour.

The records had a sampling frequency (fs) of $200 \mathrm{~Hz}$ and an anti-aliasing filter with a high cut-off frequency of $100 \mathrm{~Hz}$ was used. A major problem of EHG analysis is to design a signal conditioning, because the parameters of this phase space are strictly determined by the characteristics of the measured signal (Graczyk et al., 1998). This is important especially for analysis of uterine electrical activity in normal and complicated labour (Euliano et al., 2009).

Part of the exemplary, raw signal (containing the 1000 samples) is shown in Figure 1. In order to have the best knowledge about the information embedded in the vector of the analysed signals, we should use raw signals, without any preprocessing. In Radomski's earlier publications, (2014, 2015) it has been proved that differentiated signals are better for the analysis of EHG signals. Part of the exemplary, differentiated signal (containing the 1000 samples) is shown in Figure 2.

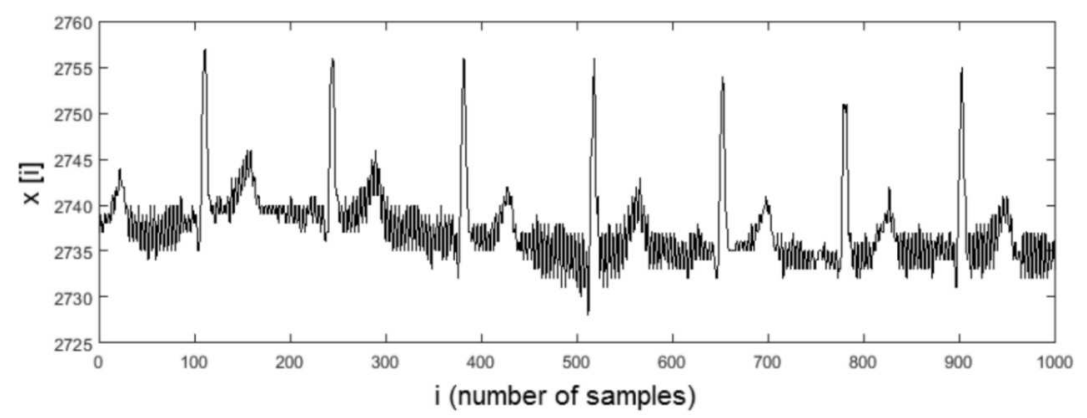

Figure 1. Raw EHG signal

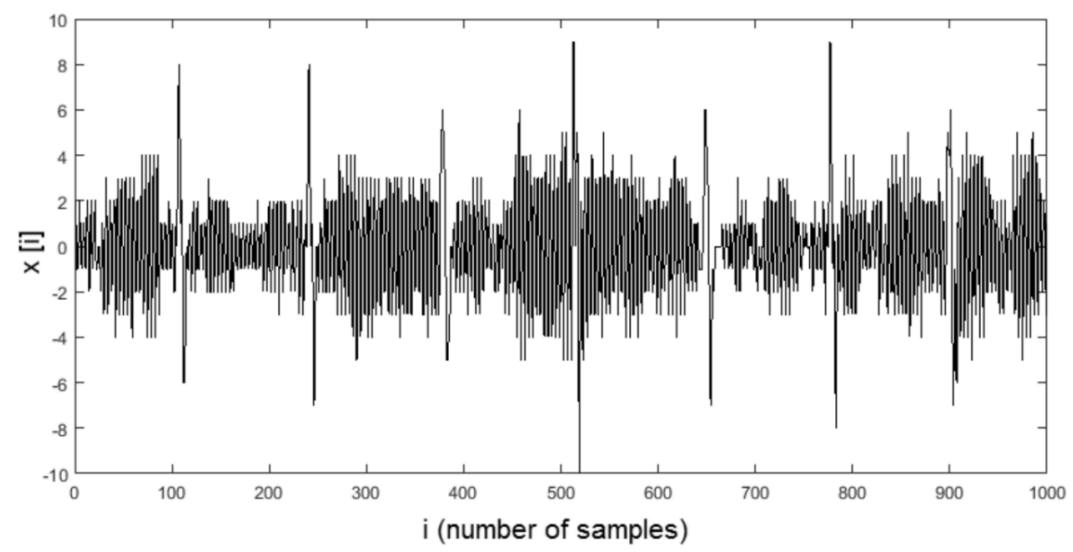

Figure 2. Differentiated EHG signal 
The signals were analysed by the Cross Recurrence Plot (CRP) Toolbox for Matlab, shared by Marwan (2014). At the beginning of phase space reconstruction, time delay was calculated by using mutual information first, for the raw signal (Figure 3a) and second, for the differentiated signal (Figure 3b). For both signals, the "number of beans" was determined based on an amount range of classes depending on the analysed signal sample amounts taken for analysis (Legg et al., 2007), as described by the statistical equation: $N o B=1+3.3 \cdot \log (n)$, where: $n$ is length of data series $(n=1000$, $N o B=11)$.

We have received time delay equals 2 for the raw signal and 1 for differentiated signal (first minimum of the mutual information).
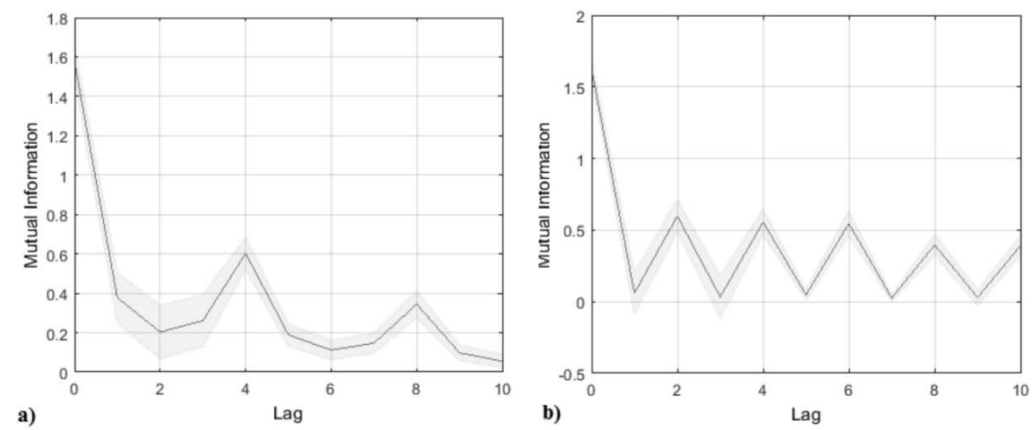

Figure 3. Mutual information for the EHG signal:

a) the raw, b) the differentiated

The next step of phase space reconstruction process was to estimate the embedding dimension. For this purpose, we used the false nearest neighbours method. For the time delay, previously obtained values were adopted as follows: for the raw signal $\tau=2$, for the differentiated signal $\tau=1$. As for the other parameters: neighbourhood criterion $R=10$, the size of neighbourhood $S=i n f$, random samples $N=1000$ (for the entire signal) and the Euclidean norm was chosen. We found the embedding dimension to equal approximately 9 for both signals - the raw (Figure $4 \mathrm{a}$ ) and the differentiated (Figure 4b).

For comparison of the results and effectiveness of Marwan's FNN method to determine the embedding dimension, the Cao criterion was used. As a result, the same values as for FNN - 3 were obtained for both signals (Figure $5 \mathrm{a}$ and $5 \mathrm{~b}$ ). This may prove the correctness of calculations in the embedding dimension for this EHG signal.

Once we have these parameters $(d, \tau)$ we can reconstruct the attractor embedded in a particular phase space. Reconstructed attractors for the raw (Figure 6a) and differentiated (Figure 6b) datasets are shown below. 
Selection of Phase Space Reconstruction Parameters for EMG Signals...
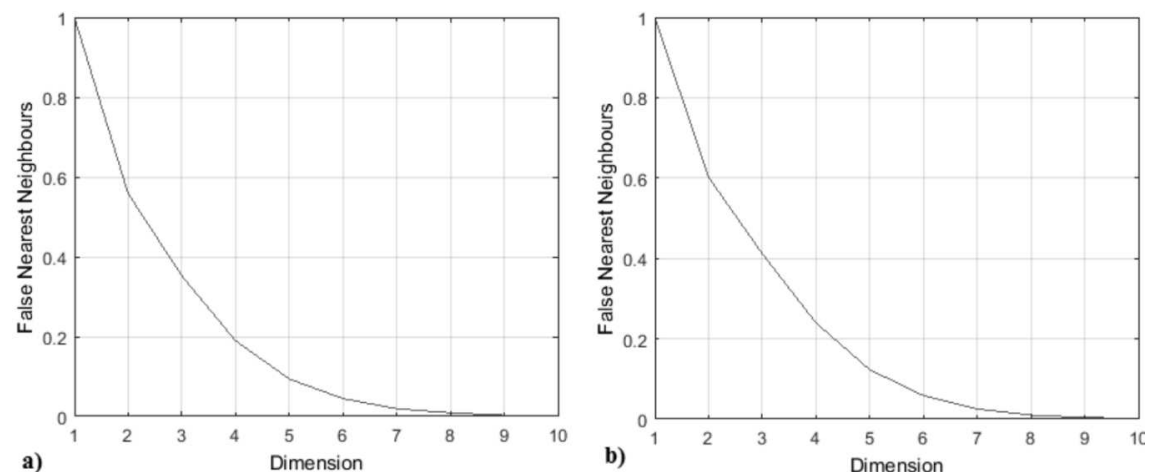

Figure 4. False nearest neighbours for the EHG signal:

a) the raw, b) the differentiated
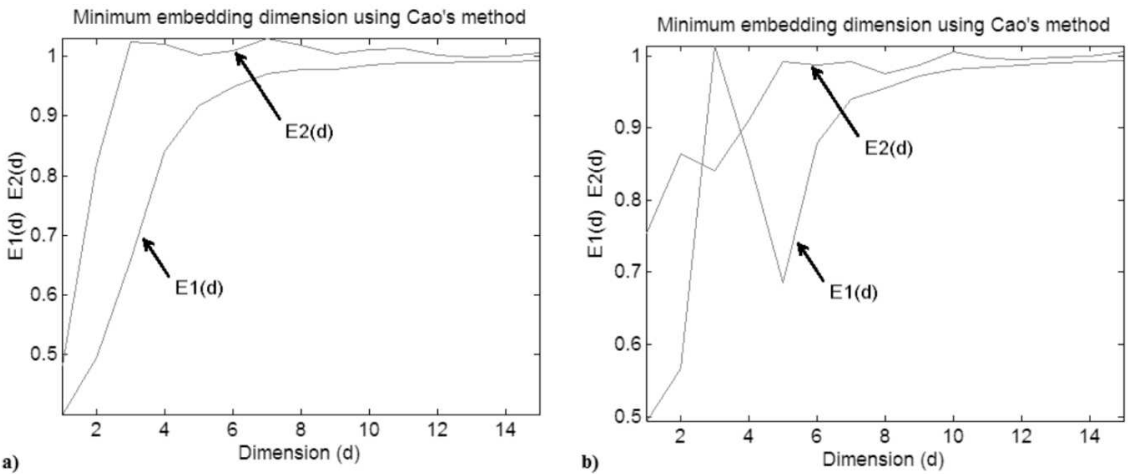

Figure 5. Minimum embedding dimension using Cao's method for the EHG signal: a) the raw, b) the differentiated
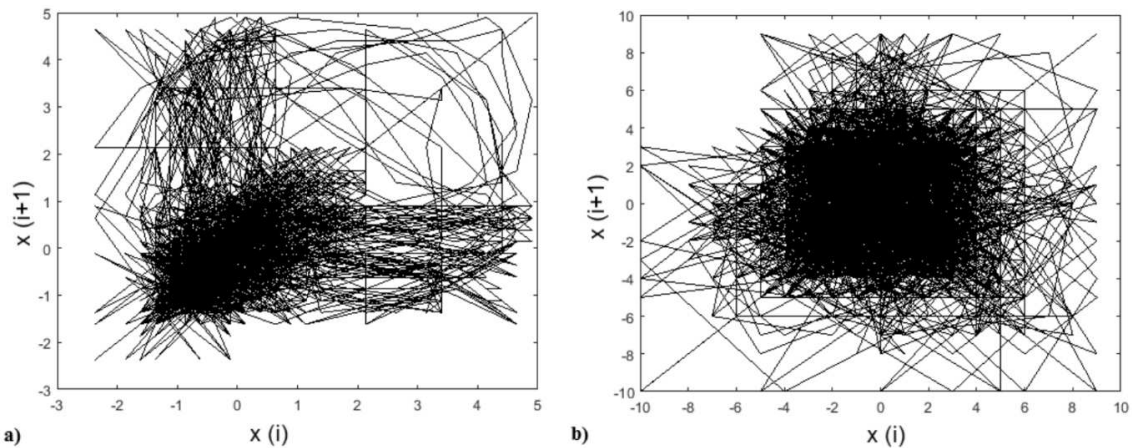

Figure 6. Phase space reconstruction for the EHG signal:

a) the raw, b) the differentiated 
In an analogous way, all the signals contained in the database were analysed. After analysis, it was found that the amplitude of contractions, their frequency, the chosen channel, and the condition of the patient's gestation (labor or pregnancy) had no significant impact on the calculated time delay and embedding dimension.

\section{Conclusions}

The choice of the embedding dimension $d$ and the time delay $\tau$ is important for nonlinear data analysis. Both parameters are tightly correlated - firstly, one needs to calculate time delay $\tau$ to be able to calculate embedding dimension $d$ in relation to its value. This is the first step in the analysis of the nonlinearity of a data set. Spontaneous depolarisation and repolarisation due to ionic currents - the main properties of smooth muscle cells in the uterus - are manifested as an electrohysterogram (EHG). Electrohysterograms of signals are dependent on patients and there is a need to develop methods to better investigate and analyse EHG signals. For reliable application of electrohysterography in obstetrics, there is a need to find a method for examination of its nonlinear properties. After the reconstruction of the phase space formed by this data, wider quantitative and qualitative analysis could be carried out. The process of reconstructing phase space is not very complicated if it is performed using the method presented in this paper.

\section{Acknowledgments}

This paper was supported by statutory funds S/WM/1/2014 from the Bialystok University of Technology and the financial support by Faculty of Mechanical Engineering, Bialystok University of Technology (MB/WM/2/2016).

\section{R E F E R E N C E S}

Abarbanel, H. D., Brown, R., Sidorowich, J. J., \& Tsimring, L. S. (1993). The analysis of observed chaotic data in physical systems. Reviews of Modern Physics, 65(4), 1331.

Alamedine, D., Diab, A., Muszynski, C., Karlsson, B., Khalil, M., \& Marque, C. (2014). Selection algorithm for parameters to characterize uterine EHG signals for the detection of preterm labor. Signal, Image and Video Processing, $8(6), 1169-1178$. 
Albano, A., Muench, J., Schwartz, C., Mees, A., \& Rapp, P. (1988). Singular-value decomposition and the Grassberger-Procaccia algorithm. Physical Review A, $38(6), 3017$.

Albano, A., Passamante, A., \& Farrell, M. E. (1991). Using higher-order correlations to define an embedding window. Physica D: Nonlinear Phenomena, 54(1-2), 85-97.

Alexandersson, A., Steingrimsdottir, T., Terrien, J., Marque, C., \& Karlsson, B. (2015). The Icelandic 16-electrode electrohysterogram database. Scientific Data, 2, 150017.

Bassingthwaighte, J. B., Liebovitch, L. S., \& West, B. J. (2013). Fractal physiology. Springer.

Broomhead, D., \& Jones, R. (1989). Time-series analysis. Paper presented at the Proceedings of the Royal Society of London A: Mathematical, Physical and Engineering Sciences.

Buzug, T., \& Pfister, G. (1992a). Comparison of algorithms calculating optimal embedding parameters for delay time coordinates. Physica D: Nonlinear Phenomena, 58(1), 127-137.

Buzug, T., \& Pfister, G. (1992b). Optimal delay time and embedding dimension for delay-time coordinates by analysis of the global static and local dynamical behavior of strange attractors. Physical Review A, 45(10), 7073-7084.

Cao, L. (1997). Practical method for determining the minimum embedding dimension of a scalar time series. Physica D: Nonlinear Phenomena, 110(1), 43-50.

Casdagli, M., Eubank, S., Farmer, J. D., \& Gibson, J. (1991). State space reconstruction in the presence of noise. Physica D: Nonlinear Phenomena, 51(1), $52-98$.

Chen, M., Fang, Y., \& Zheng, X. (2014). Phase space reconstruction for improving the classification of single trial EEG. Biomedical Signal Processing and Control, 11, 10-16.

Diab, A., Falou, O., Hassan, M., Karlsson, B., \& Marque, C. (2015). Effect of filtering on the classification rate of nonlinear analysis methods applied to uterine EMG signals. Paper presented at the 37th Annual International Conference of the IEEE Engineering in Medicine and Biology Society (EMBC).

Diab, A., Hassan, M., Marque, C., \& Karlsson, B. (2012). Quantitative performance analysis of four methods of evaluating signal nonlinearity: application to uterine EMG signals. Paper presented at the Annual International Conference of the IEEE Engineering in Medicine and Biology Society.

Erem, B., Orellana, R. M., Hyde, D. E., Peters, J. M., Duffy, F. H., Stovicek, P., Warfield, S. K., et al. (2016). Extensions to a manifold learning framework for time-series analysis on dynamic manifolds in bioelectric signals. Physical Review E, 93(4), 042218. 
Euliano, T. Y., Marossero, D., Nguyen, M. T., Euliano, N. R., Principe, J., \& Edwards, R. K. (2009). Spatiotemporal electrohysterography patterns in normal and arrested labor. American Journal of Obstetrics and Gynecology, 200(1), 54.e1-54.e7.

Fraser, A. M., \& Swinney, H. L. (1986). Independent coordinates for strange attractors from mutual information. Physical Review A, 33(2), 1134.

Goldberger, A. L., Amaral, L. A., Glass, L., Hausdorff, J. M., Ivanov, P. C., Mark, R. G., Mietus, et al. (2000). Physiobank, physiotoolkit, and physionet. Components of a new research resource for complex physiologic signals. Circulation, 101(23), e215-e220.

Graczyk, S., Horoba, K., Jerewski, J., Wrobel, J., \& Gacek, A. (1998). Use of running statistical evaluation in analysis of electrohysterographic signals. Paper presented at the 8th Proceedings of the Mediterranean Conference on Medical and Biological Engineering and Computing.

Grassberger, P., \& Procaccia, I. (1983). Characterization of strange attractors. Physical Review Letters, 50(5), 346-349.

Huffaker, R. (2010). Phase Space Reconstruction from Time Series Data: Where History Meets Theory. Proceedings in System Dynamics and Innovation in Food Networks 2010, 1-9.

Jaśkowski, P. (1995). Zastosowanie metod dynamiki nieliniowej do analizy sygnału EEG człowieka. Current Topics in Biophysics, 19, 42-57.

Kliková, B., \& Raidl, A. (2011). Reconstruction of phase space of dynamical systems using method of time delay. WDS'11 Proceedings of Contributed Papers: Part III - Physics, pp. 83-87.

Korus, L., \& Piorek, M. (2015). Compound method of time series classification. Nonlinear Analysis, Modelling and Control, 20(4), 545-560.

Lainscsek, C., \& Sejnowski, T. J. (2015). Delay differential analysis of time series. Neural computation, 27(3), 594-614.

Legg, P. A., Rosin, P. L., Marshall, D., \& Morgan, J. E. (2007). Improving accuracy and efficiency of registration by mutual information using Sturges histogram rule. Paper presented at the 11th Annual Conference in Medical Image Understanding and Analysis (pp. 26-30).

Liebert, W., Pawelzik, K., \& Schuster, H. (1991). Optimal embeddings of chaotic attractors from topological considerations. EPL (Europhysics Letters), 14(6), $521-526$.

Marwan, N. (2014). Cross Recurrence Plot Toolbox for Matlab, Ver.5.15, Release 28.10 .2015

Ouyang, G., \& Li, X. (2016). Dynamical Similarity Analysis of EEG Recordings Signal Processing in Neuroscience (pp. 111-124). Singapore: Springer.

Packard, N. H., Crutchfield, J. P., Farmer, J. D., \& Shaw, R. S. (1980). Geometry from a Time Series. Physical Review Letters, 45(9), 712. 
Palit, S. K., Mukherjee, S., Banerjee, S., Ariffin, M., \& Bhattacharya, D. (2015). Some Time-Delay Finding Measures and Attractor Reconstruction Applications of Chaos and Nonlinear Dynamics in Science and Engineering - Vol. 4 Understanding Complex Systems (pp. 215-256). Switzerland: Springer.

Piórek, M. (2016). Mutual Information for Quaternion Time Series. Paper presented at the IFIP International Conference on Computer Information Systems and Industrial Management.

Pritchard, W. S., \& Duke, D. W. (1995). Measuring chaos in the brain - a tutorial review of EEG dimension estimation. Brain and Cognition, 27(3), 353-397.

Przybyła, T., Pander, T., Wróbel, J., Czabański, R., Roj, D., \& Matonia, A. (2014). $A$ recovery of FHR signal in the Embedded Space. Paper presented at the XIII Mediterranean Conference on Medical and Biological Engineering and Computing.

Radomski, D. S. (2014). A mulltivariate sample entropy of differentiated electtrohysterographical signals for an identification of an Uterine Labor Activity. Information Technology in Biomedicine, 4, 303-310.

Radomski, D. S. (2015). A nonlinear parameterization of multivariate electrohysterographical signals. Computers in Biology and Medicine, 67, 13-20.

Ruelle, D. (1990). The Claude Bernard Lecture, 1989. Deterministic chaos: the science and the fiction. Paper presented at the Proceedings of the Royal Society of London A: Mathematical, Physical and Engineering Sciences.

Takens, F. (1981). Detecting strange attractors in turbulence. Dynamical systems and turbulence, Warwick 1980 (pp. 366-381). Springer-Verlag Berlin Heidelberg.

Xia, D.-H., Song, S.-Z., \& Behnamian, Y. (2016). Detection of corrosion degradation using electrochemical noise (EN): review of signal processing methods for identifying corrosion forms. Corrosion Engineering Science and Technology, 51(7), 527-544. 